\title{
Erratum to: Atomic and molecular decompositions of anisotropic Besov spaces
}

\author{
Marcin Bownik
}

Published online: 2 February 2013

(C) Springer-Verlag Berlin Heidelberg 2013

\section{Erratum to: Math Z (2005) 250:539-571 DOI 10.1007/s00209-005-0765-1}

We give a corrected proof of Lemma 3.1 in [1].

While the statement of [1, Lemma 3.1] is true, its proof is incorrect. The argument contains a serious defect which can not be easily corrected. The inequality that appears in [1] before (3.5) is not true. If this inequality was true, then we could conclude that, even for a non doubling measure $\mu,(3.5)$ was also true. But there exist some non doubling measures for which (3.5) is not true. Since this result plays a fundamental role in the rest of the paper, it becomes compulsory to provide the correct proof of [1, Lemma 3.1].

Lemma 1.1 Suppose $K$ is a compact subset of $\mathbb{R}^{n}, 0<p<\infty$, and $\mu$ is a $\rho_{A}$-doubling measure on $\mathbb{R}^{n}$ with respect to some expansive dilation A. Suppose $f \in \mathcal{S}^{\prime}\left(\mathbb{R}^{n}\right)$ and $\operatorname{supp} \hat{f} \subset$ $\left(A^{*}\right)^{j} K$ for some $j \in \mathbb{Z}$. Then

$$
\left(\sum_{k \in \mathbb{Z}^{n}} \sup _{z \in Q_{j, k}}|f(z)|^{p} \mu\left(Q_{j, k}\right)\right)^{1 / p} \leq C\|f\|_{L^{p}(\mu)},
$$

where $Q_{j, k}=A^{-j}\left([0,1]^{n}+k\right)$, and the constant $C=C(K, p, \mu)$ depends on $K, p$, and the doubling constant of $\mu$.

Proof We claim that it suffices to show (1.1) only for $j=0$. Let $f \in \mathcal{S}^{\prime}\left(\mathbb{R}^{n}\right)$ be such that supp $\hat{f} \subset\left(A^{*}\right)^{j} K$ for some $j \in \mathbb{Z}$. Since $f$ is a regular distribution, i.e., $f$ is identified with

The author wishes to thank Begoña Barrios Barrera for pointing out to the mistake in the proof of Lemma 3.1 in [1].

The online version of the original article can be found under doi:10.1007/s00209-005-0765-1.

M. Bownik ( $\square)$

Department of Mathematics, University of Oregon, Eugene, OR 97403-1222, USA

e-mail: mbownik@uoregon.edu 
some locally integrable function, we can define a dilate $g \in \mathcal{S}^{\prime}\left(\mathbb{R}^{n}\right)$ of $f$ by $g(x)=f\left(A^{-j} x\right)$. Then, the support of the distribution $\hat{g}$ satisfies

$$
\operatorname{supp} \hat{g}=\left(A^{*}\right)^{-j}(\operatorname{supp} \hat{f}) \subset K \text {. }
$$

Let $\mu_{j}$ be a dilate of a measure $\mu$ given by $\mu_{j}(E)=\mu\left(A^{-j} E\right)$ for Borel subsets $E \subset \mathbb{R}^{n}$. Observe that $\mu_{j}$ has the same doubling constant as $\mu$. Assuming that (1.1) holds for $j=0$ we have

$$
\left(\sum_{k \in \mathbb{Z}^{n}} \sup _{z \in Q_{0, k}}|g(z)|^{p} \mu_{j}\left(Q_{0, k}\right)\right)^{1 / p} \leq C\|g\|_{L^{p}\left(\mu_{j}\right)} .
$$

Observe that $\mu_{j}\left(Q_{0, k}\right)=\mu\left(A^{-j}\left(Q_{0, k}\right)\right)=\mu\left(Q_{j, k}\right)$. Moreover,

$$
\sup _{z \in Q_{0, k}}|g(z)|^{p}=\sup _{z \in Q_{0, k}}\left|f\left(A^{-j} z\right)\right|^{p}=\sup _{z \in Q_{j, k}}|f(z)|^{p} .
$$

Finally, by the change of variables

$$
\int_{\mathbb{R}^{n}}|g(x)|^{p} d \mu_{j}(x)=\int_{\mathbb{R}^{n}}\left|f\left(A^{-j} x\right)\right|^{p} d \mu_{j}(x)=\int_{\mathbb{R}^{n}}|f(x)|^{p} d \mu(x) .
$$

Combining the above with (1.2) yields (1.1).

To deal with the case $j=0$ in (1.1) we shall apply [2, Lemma 8.3] which is an adaption of Peetre's mean value inequality [3, Lemma A.4]. Note that the proof of this result in [2] is self-contained and does not depend on any conclusions drawn from [1, Lemma 3.1]. Let $\mathcal{Q}=\left\{Q_{j, k}: j \in \mathbb{Z}, k \in \mathbb{Z}^{n}\right\}$. For $Q=Q_{j, k} \in \mathcal{Q}$ we denote $\operatorname{scale}(Q)=-j$ and $x_{Q}=A^{-j} k$. For any $Q \in \mathcal{Q}$ we define

$$
a_{Q}=\sup _{y \in Q}|f(y)|, \quad b_{Q}=\sup \left\{\inf _{y \in P}|f(y)|: \operatorname{scale}(P)=\operatorname{scale}(Q)-\gamma, P \cap Q \neq \emptyset\right\} .
$$

For any $r, \lambda>0$ define a majorant sequence

$$
\left(a_{r, \lambda}^{*}\right)_{Q}=\left(\sum_{P \in \mathcal{Q}_{0}} \frac{\left|a_{P}\right|^{r}}{\left(1+\rho_{A}\left(x_{Q}-x_{P}\right)\right)^{\lambda}}\right)^{1 / r} .
$$

Likewise we define $\left(b_{r, \lambda}^{*}\right)_{Q}$. Then, [2, Lemma 8.3] says that there exists $\gamma \in \mathbb{N}$ such that

$$
\left(a_{r, \lambda}^{*}\right)_{Q} \asymp\left(b_{r, \lambda}^{*}\right)_{Q} \text { for all } Q \in \mathcal{Q}_{0}:=\left\{Q_{0, k}: k \in \mathbb{Z}^{n}\right\},
$$

with constants independent of $f$ and $Q$. By [2, (2.6)] we have

$$
\mu(Q) \leq C\left(1+\rho_{A}\left(x_{P}-x_{Q}\right)\right)^{\beta} \mu(P) \text { for } P, Q \in \mathcal{Q}_{0},
$$

where $\beta>1$ is the doubling constant of $\mu$.

We shall apply (1.3) when $r=p$ and $\lambda>\beta+1$. By (1.3) and (1.4)

$$
\begin{aligned}
\sum_{Q \in \mathcal{Q}_{0}}\left|a_{Q}\right|^{p} \mu(Q) & \leq C \sum_{Q \in \mathcal{Q}_{0}}\left|\left(b_{p, \lambda}^{*}\right)_{Q}\right|^{p} \mu(Q) \leq C \sum_{Q \in \mathcal{Q}_{0}} \sum_{P \in \mathcal{Q}_{0}} \frac{\left|b_{P}\right|^{p}}{\left(1+\rho_{A}\left(x_{Q}-x_{P}\right)\right)^{\lambda}} \mu(Q) \\
& \leq C \sum_{P \in \mathcal{Q}_{0}}\left|b_{P}\right|^{p} \mu(P) \sum_{Q \in \mathcal{Q}_{0}} \frac{1}{\left(1+\rho_{A}\left(x_{Q}-x_{P}\right)\right)^{\lambda-\beta}} \leq C \sum_{P \in \mathcal{Q}_{0}}\left|b_{P}\right|^{p} \mu(P) .
\end{aligned}
$$


In the last step we used the fact that $\sum_{k \in \mathbb{Z}^{n}}\left(1+\rho_{A}(k)\right)^{-1-\varepsilon}<\infty$ for $\varepsilon>0$. Hence,

$$
\begin{aligned}
\int_{Q}|f(x)|^{p} d \mu(x) & \geq \sum_{P \in \mathcal{Q}, \operatorname{scale}(P)=-\gamma} \int_{P \cap Q}|f(x)|^{p} d \mu(x) \\
& \geq \sum_{P \in \mathcal{Q}, \operatorname{scale}(P)=-\gamma} \inf _{z \in P}|f(z)|^{p} \mu(P \cap Q) \\
& \geq \sum_{P \in \mathcal{Q}, \operatorname{scale}(P)=-\gamma}\left|b_{Q}\right|^{p} \mu(P \cap Q)=\left|b_{Q}\right|^{p} \mu(Q) .
\end{aligned}
$$

Summing the above over $Q \in \mathcal{Q}_{0}$ and combining with (1.5) yields

$$
\sum_{Q \in \mathcal{Q}_{0}}\left|a_{Q}\right|^{p} \mu(Q) \leq C \sum_{Q \in \mathcal{Q}_{0}} \int_{Q}|f(x)|^{p} d \mu(x)=\|\left. f\right|_{L^{p}(\mu)} ^{p} .
$$

This completes the proof of Lemma 1.1.

\section{References}

1. Bownik, M.: Atomic and molecular decompositions of anisotropic Besov spaces. Math. Z. 250, 539-571 (2005)

2. Bownik, M.: Anisotropic Triebel-Lizorkin spaces with doubling measures. J. Geom. Anal. 17, 387-424 (2007)

3. Frazier, M., Jawerth, B.: A discrete transform and decomposition of distribution spaces. J. Funct. Anal. 93, 34-170 (1990) 